\title{
TRANSPORTE COOPERATIVO DE OBJETOS CON UNA PLATAFORMA MÓVIL EN UN ENTORNO ESTRUCTURADO
}

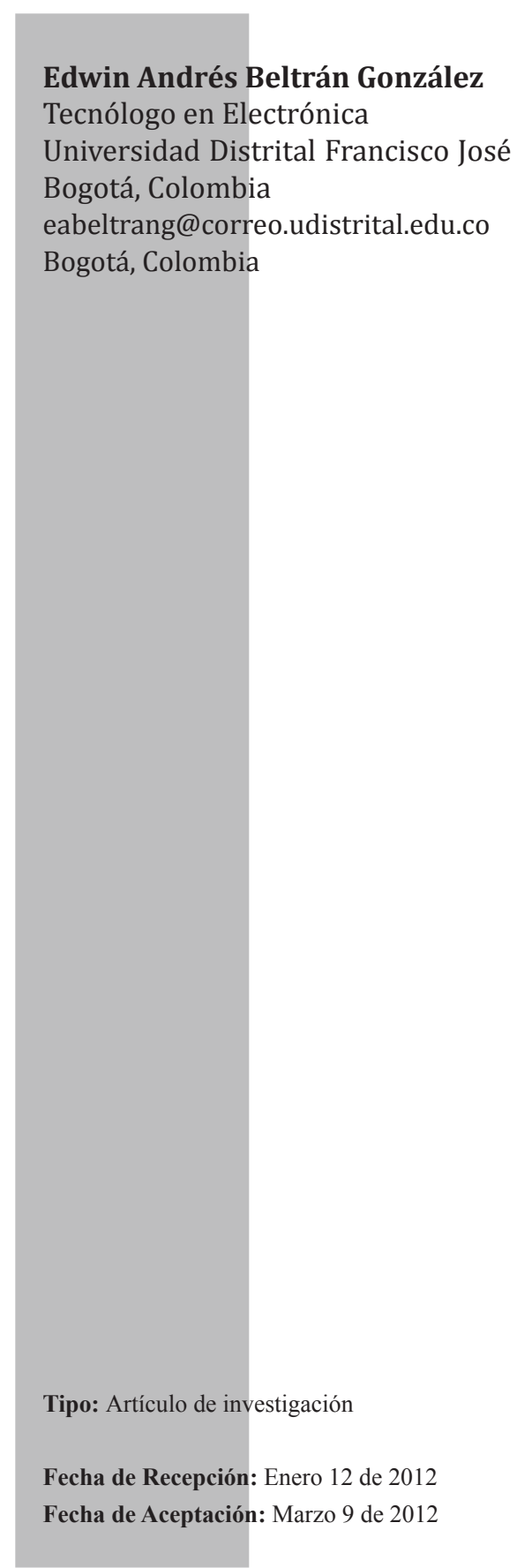

\section{COOPERATIVE TRANSPORT OF OBJECTS USING A MOBILE PLATFORM WITHIN A STRUCTURED ENVIRONMENT}

\begin{abstract}
Cooperative robotics is intended to design systems that consist of various robots capable of solving problems jointly. Therefore, implementations of this nature might require various types of control (either centralized or distributed). Based on these ideas, object transport has become one of the challenges within this research field. The point is to implement various strategies ranging from pulling objects to actually lifting them or dragging them. These types of tasks have been addressed since the 90s up until recently. The present work attempts to show the development and implementation of a cooperative robotic platform applied to large-object transport by means of a structured environment, using the tools provided by the LEGO MINDSTORMS RCX 2.0 kit and some other tools belonging to a research group called ROMA (Robótica Móvil Autónoma).
\end{abstract}

Key words: cooperative robotics, robotic systems, multi-agent, coordination, communication.

\section{RESUMEN}

La robótica cooperativa busca diseñar sistemas compuestos de varios robots capaces de resolver problemas conjuntamente, de tal manera que dicha implementación puede llevar consigo distintos tipos de control (centralizado o distribuido), de esta manera una de las aplicaciones en esta línea de investigación es el transporte de objetos mediante distintas estrategias que van desde el empuje, la sujeción y arrastre de los objetos; tales trabajos se han presentado desde la década de los 90 hasta el día de hoy. El enfoque del presente artículo busca mostrar el desarrollo e implementación de una plataforma robótica cooperativa aplicada al transporte de objetos largos a través de un entorno estructurado utilizando las herramientas que provee el kit Lego Mindstorms RCX 2.0 y el grupo de investigación en robótica móvil autónoma ROMA.

Palabras claves: robótica cooperativa, sistemas robóticos multiagente, coordinación, comunicación. 


\section{INTRODUCCIÓN}

En la actualidad los desarrollos en el campo de la robótica están centrados en el diseño de sistemas robóticos multiagente para aplicaciones que van desde la navegación en entornos estructurados y poco estructurados hasta el transporte cooperativo de objetos.

Dentro del grupo de investigación en robótica móvil autónoma (ROMA) estamos conscientes de la necesidad de explorar el campo de la robótica cooperativa y así estar a la vanguardia a nivel mundial, por tal motivo el objetivo de este artículo es mostrar los resultados obtenidos con el diseño e implementación de una plataforma móvil aplicada en trabajo cooperativo para el transporte de objetos dentro de un escenario estructurado utilizando las herramientas que ofrece el kit Lego Mindstorms RCX 2.0® [1].

\subsection{Sistemas robóticos multiagente}

Un sistema multi-robot [2], [3] se define como un conjunto de robots situados en un entorno común pero que no necesariamente poseen capacidades para interactuar con otros robots o presentan un comportamiento proactivo; por ejemplo un conjunto de robots seguidores de línea sobre un tablero común puede ser catalogado como un sistema multirobot, sin embargo estos últimos no cumplen las características identificadas en [4], [5] y solo aquellos prototipos que cumplan estas características pueden ser denominados como agentes robóticos [6], [10].

Por lo general cuando se habla de sistemas multi-robot, existen dos categorías generales para abordar la temática: los sistemas de enjambres colectivos y los sistemas intencionalmente cooperativos.

Los sistemas de enjambres colectivos son aquellos en los que los robots ejecutan sus tareas con un conocimiento mínimo o nulo acerca del estado o progreso de los demás robots del equipo, por lo general esta concepción está basada en el principio de que un grupo numeroso de robots comunicándose mínimamente puede generar comportamientos colectivos a gran escala. Por otra parte están los sistemas intencionalmente cooperativos en los cuales cada robot esta finamente ligado a los demás y constantemente sabe la ubicación, estado, creencias e información de cada uno de los integrantes del equipo, de esta manera todos apuntan hacia una misma meta, sus protocolos de comunicación permiten establecer dos tipos de solución. Dentro de ellos la primera es una solución fuertemente cooperativa donde cada uno de los robots hace parte de una sola unidad mayor, en la cual todos los robots apuntan hacia una misma tarea y cada uno aporta sus capacidades individuales para mejorar las capacidades globales. La segunda es una solución débilmente cooperativa en donde los robots tienen ciertos tiempos de independencia y pueden realizar las acciones que vean convenientes pero que a la hora de evaluar un resultado global no aportan a la solución del problema general [11].

Los sistemas multi-robot son también llamados sistemas robóticos multiagente [2], debido a que sus categorías involucran implícitamente el concepto de robot agente [6], [10], así, para poder llevar a cabo una mínima interacción cada autómata debe percibir su medio, poseer mecanismos de decisión de acciones y desde luego un conocimiento de la evolución del medio con las acciones realizadas por él y por los integrantes del equipo.

Un primer término que se debe explorar a la hora de hablar de sistemas robóticos multiagente es el de "coordinación" que se define [12], [13] como la propiedad que tiene un conjunto de agentes para realizar alguna actividad colectiva; el grado de coordinación exhibido por un conjunto de agentes es el área en que se pueden evitar para realizar un trabajo articulado. Una coordinación efectiva implica un grado de predictibilidad mutua y cuantos más conflictos inesperados se den, peor están coordinados los agentes robot.

De lo anterior se desprende la definición de "cooperación" que según [10], [14] es una forma de interacción mediante comunicación 
directa [15] en la cual el sistema robótico multiagente presenta un comportamiento de trabajo colaborativo en aras de conseguir una meta común consiguiendo resultados de manera más efectiva en un menor tiempo.

El incremento en el desempeño del sistema robótico multiagente evidencia un comportamiento meramente cooperativo, para lo cual el mecanismo de control puede estar basado en la imposición por parte de diseñador de una o varias estructuras de control que generen las dinámicas de interacción de los comportamientos presentados por los agentes robot.

\section{TRABAJOS REALIZADOS ALREDEDOR DEL TRANSPORTE COOPERATIVO}

Una de las aplicaciones del trabajo cooperativo es el transporte de objetos que muchas veces suele ser un problema para un solo robot, mediante la utilización de múltiples mecanismos como sujeción, truncamiento o simplemente arrastre, a continuación se muestran algunos trabajos alrededor del tema. Mataric et al [16] diseño una plataforma cooperativa meramente reactiva aplicada al transporte de una caja larga, su enfoque utilizo la cooperación en tres niveles: sensado, acción y control, para la distribución de tareas entre dos robots hexápodos equipados con sensores de luz y de contacto que les permitía generar comportamientos de sujeción y seguimiento de luz dependiendo del estado de la tarea.

Donalds et al [17] desarrollo un sistema multirobot descentralizado cooperativo enfocado al movimiento de un grupo de cajas desordenadas, su diseño consistía en el desarrollo de tres habilidades. La primera consistía en lanzar una cuerda alrededor de los objetos con la intención de sujetarlos. La segunda radicaba en la utilización de una cuerda floja para acomodar las cajas internamente. Por último el sistema tenía la capacidad de transportar las cajas a acomodadas a otro sitio asegurando su orden con la implementación de trinquetes sobre las cajas.
Kube [18], [19] presento el primer modelo formal para transporte cooperativo cuyo enfoque fue la resolución de problemas descentralizadamente. En este trabajo el autor muestra que si el tamaño del objeto es más pequeño respecto al sistema de sujeción de los robots el desempeño de la plataforma decrece drásticamente ya que la superficie de contacto es limitada.

Wang et al [20] desarrollo un sistema multirobot enfocado en el transporte de objetos cooperativamente para lo cual utilizo una estrategia que le permitía atrapar o encerrar el objeto con los robots a través del diseño de algoritmos descentralizados basados en campos de potencial que le permitieron a la plataforma mantener el objeto atrapado simultáneamente de irlo arrastrando hasta una meta deseada.

Gross et al [21], [22] diseño un sistema completamente descentralizado en el marco del proyecto Swarm-bots [23], [24] para el transporte de objetos pesados y de distintos tamaños y formas, la plataforma estaba compuesta por 16 robots autónomos con capacidades de auto ensamblaje llamados s-bots. Estos a su vez eran capaces de establecer una conexión física con cada objeto a transportar. Cada robot era controlado mediante algoritmos evolutivos $y$ redes neuronales, lo que les permitía adaptarse a cualquier entorno sin la necesidad de conocer su posición inicial, también les ofrecía la ventaja de calcular y determinar la geometría del objeto y con esto aumentar o disminuir el número de s-bots para transportar el objeto.

En el marco del proyecto Swarmanoid [25], [26] Decugniere et al [27] presentó un enfoque para el transporte cooperativo de múltiples objetos por un sistema de enjambre robótico con el fin de aumentar su capacidad de carga inspirado por las observaciones hechas al transporte de alimentos en colonias de insectos. Su propuesta radica en la implementación de un robot que actúa como un vehículo de carga masiva al que denomino cart-bot en el cual se apilaba uno a uno los objetos para luego ser llevado por otros 
robots denominados foot-bots hasta una meta deseada.

Dorigo et al [28], [30] retoma todo el trabajo realizado por Gross y Decugniere y añade a este la implementación de algoritmos evolutivos y redes neuronales para la detección y prevención de obstáculos, el nivel de dificultad radico en la generación de comportamientos que le permitieran al robot prevenir en obstáculo y a la vez mantener el transporte del objeto.

\section{METODOLOGÍA}

Se diseño e implemento una plataforma móvil cooperativa aplicada al transporte de objetos largos utilizando las herramientas del kit Lego Mindstorms RCX 2.0® [1], a continuación se muestra el desarrollo de la investigación.

\subsection{Desarrollo del entorno}

El diseño y elaboración de un entorno conocido, le permitió a la plataforma percibir su medio eficazmente, inicialmente fue diseñado $y$ construido sobre una lamina de madera de $1 \mathrm{~m}^{2}$ de color negro mate a la cual se añadió una serie de franjas blancas (Fig. 1).

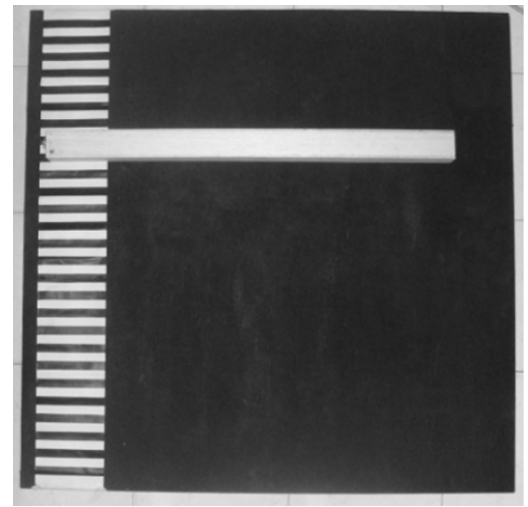

Fig. 1. Aspecto de la fase inicial del entorno estructurado.

A través del desarrollo de la investigación el entorno sufrió algunos cambios que se dieron para mejorar el desempeño de la plataforma, de esta manera el entorno en donde se realizaron las pruebas finales constaba de una lamina de madera de $1,5 \mathrm{~m}^{2}$ de color negro mate y con franjas blancas en el extremo izquierdo cuya longitud era de $0,21 \mathrm{~m}$ por $0,018 \mathrm{~m}$ de ancho (Fig. 2). Como parte del entorno, los objetos contaban de una caja de $1 \mathrm{~m}$ de longitud, una caja de $0,35 \mathrm{~m}$ de longitud y un cilindro de $1,8 \mathrm{~m}$ de longitud.

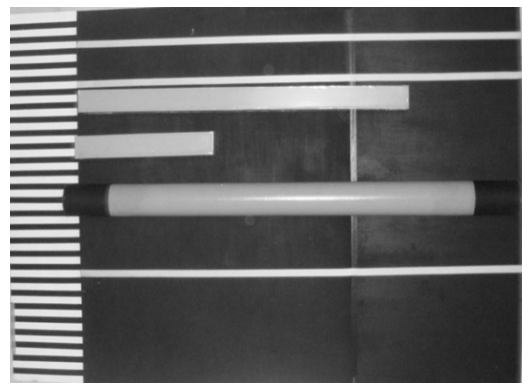

Fig. 2. Fase final del entorno estructurado.

\subsection{Desarrollo de la plataforma robótica}

Para el desarrollo de la plataforma se utilizaron las herramientas del kit Lego Mindstorms RCX 2.0® [1] diseñando un par de robots denominados maestro y esclavo cuyas características físicas permitieron arrastrar el objeto, de esta manera cada robot contó con un juego de sensores característicos del kit, así en una fase inicial, el robot maestro contaba con un sensor de contacto, y un par de sensores de rotación, mientras que para el robot esclavo se contaba con un sensor de contacto y un par de sensores de luz, el sistema de locomoción implementado por sus prestaciones fue el oruga al ofrecer mayor superficie de contacto a la hora de realizar el transporte del objeto (Fig. 3).

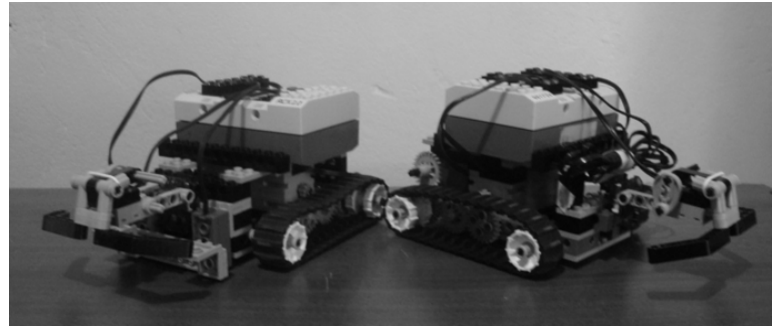

Fig. 3. Plataforma inicial para el transporte cooperativo.

\subsubsection{Sensorica}

Las exigencias de diseño y las limitaciones del kit con el que se implemento la plataforma hicieron evidente la necesidad de hacer 
una correcta aplicación de los sensores disponibles de esta manera algunos aspectos como la exploración y empuje del objeto y el mantenimiento de la referencia en cuanto a la posición fueron criterios asumidos en cuenta para cada uno de los robots.

El sensor se rotación se basa en un encoder de baja resolución, constituido por dos foto transistores y un rotor de cuatro dientes con esto se obtiene 16 pasos por vuelta, lo que traduce 22.5 grados de precisión, su implementación permitió conocer la posición del robot maestro en cada fase del proyecto y así determinar de la misma manera la posición del robot esclavo. Con el sensor de luz el robot esclavo se movilizo a través de la línea mediante la comparación del umbral de luz tanto para el color negro como para el color blanco, dicha variación permitió diseñar un comportamiento reactivo de seguidor de línea (Fig. 4).

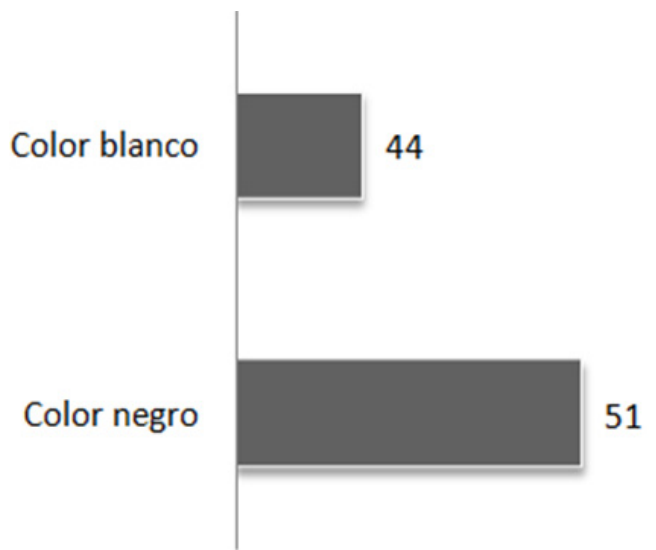

Fig. 4. Umbrales de luz obtenidos por el sensor para cada color.

\subsection{Trabajo cooperativo}

La plataforma fue diseñada para la aplicación de trabajo cooperativo al movimiento de objetos, sin embargo durante el desarrollo del proyecto se podían presentar dos situaciones, una en donde el robot maestro solicitaba ayuda al robot esclavo para mover el objeto (trabajo cooperativo), y otra en donde el robot maestro trasladaba el objeto solo (no cooperativismo).

Así, el proyecto se dividió en cuatro fases.
1. Ubicación inicial.

2. Reconocimiento del objeto.

3. Ubicación de traslado.

4. Transporte del objeto.

\subsubsection{Ubicación inicial}

En esta fase se definieron las posiciones de partida para la plataforma, los objetos y los puntos de llegada, es importante resaltar que el proyecto fue enfocado en el transporte de objetos y no de su búsqueda, de esta manera se establecieron estos puntos arbitrariamente.

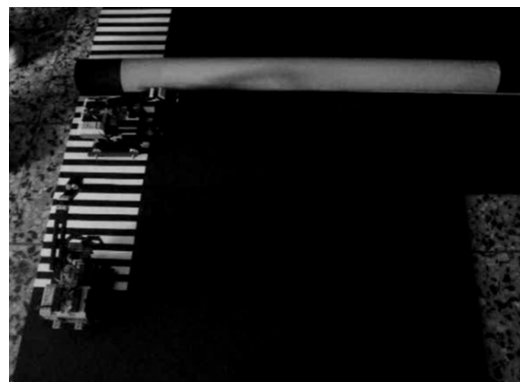

Fig. 5. Posición inicial de la plataforma y los objetos.

En la Fig. 5 se muestra la posición inicial del robot maestro, esta se indico con una línea amarilla vertical localizada en la cebra, la parte trasera del robot se ubico de forma paralela a la línea amarilla. La posición inicial del robot esclavo se implemento de tal forma que los sensores de luz estuviesen situados sobre una misma franja blanca.

\subsubsection{Reconocimiento del objeto}

El enfoque para desarrollar esta parte fue el de tocar, retroceder, avanzar de forma paralela a objeto y volver a tocar hasta no encontrar el objeto, (Fig. 6) filosofía que salvo algunos cambios se mantuvo hasta la finalización del proyecto. Muchos de los errores del proyecto se presentaron y se solucionaron en esta parte, uno de los más frecuentes fue el de lograr que el robot se mantuviera en línea recta paralela al objeto, pues aun con los sensores de rotación el control de este movimiento se dificulto bastante debido a factores como malas uniones entre la piezas de la parte de tracción de la plataforma, falta de batería, y un objeto lo suficientemente pertinente para el reconocimiento. Finalmente después de varias pruebas se obtuvieron en 
general los siguientes valores para el ángulo que describía el robot maestro tomando como referencia un movimiento de 90 grados.

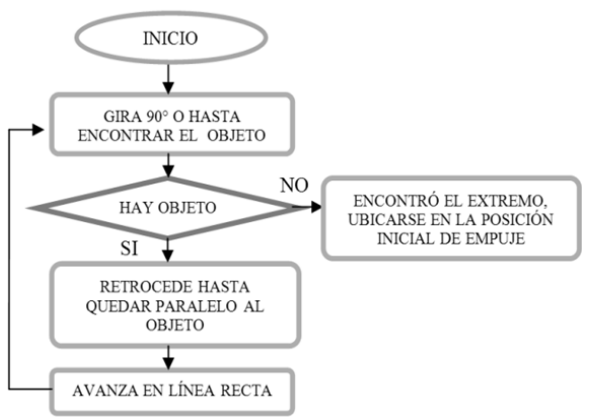

Fig. 6. Diagrama de flujo para la fase de reconocimiento del objeto realizado por el robot maestro.

\subsubsection{Ubicación de traslado}

Una vez terminada la fase de reconocimiento del objeto, el robot maestro determinaba si se establecía una situación de cooperativismo, (Fig. 7, Fig. 8) en este caso el robot esclavo partía de su posición de inicio y se ubicaba en el extremo opuesto al elegido por el agente maestro de suceder lo contrario (no cooperativismo), el robot maestro se ubicaba en la parte central del objeto y se alistaba para trasladarlo (Fig. 9).

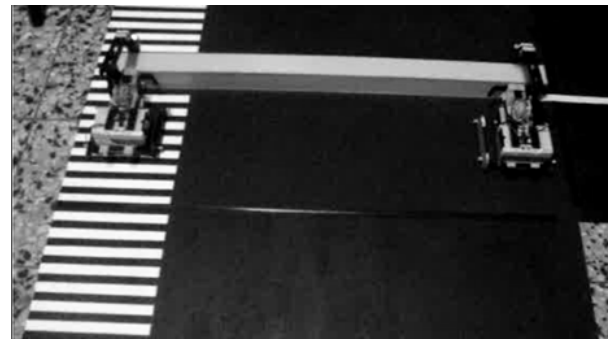

Fig. 7. Ubicación de traslado una vez establecido el transporte cooperativo sujetando una caja.

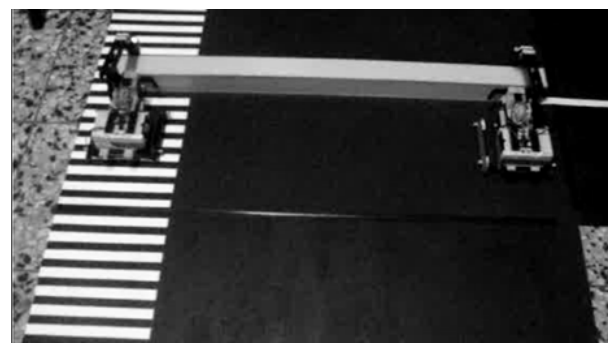

Fig. 8. Ubicación de traslado una vez establecido el transporte cooperativo sujetando un cilindro.

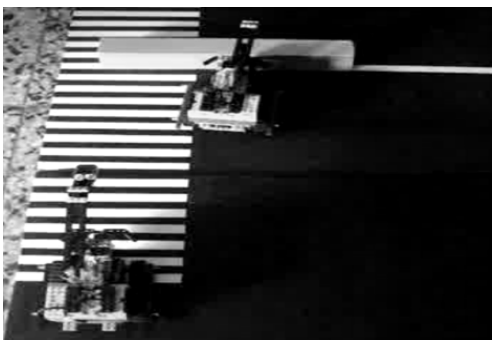

Fig. 9. Ubicación de traslado establecida para el transporte no cooperativismo.

\subsubsection{Transporte del objeto}

Una vez la plataforma estaba ubicada, el robot maestro se desplazaba hacia delante con velocidad constante, comunicándole continuamente la posición al robot esclavo (Fig. 10).

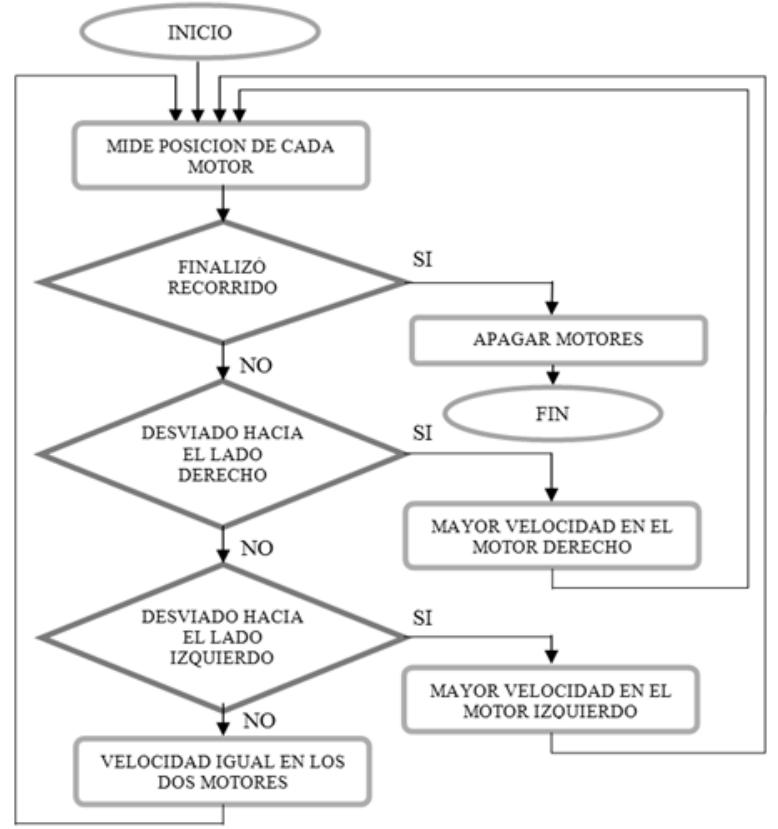

Fig. 10. Diagrama de flujo para la fase de transporte del objeto.

\subsection{Comunicación}

En la primera fase del proyecto la plataforma presentaba tiempos "muertos" en donde no había ningún tipo de interacción con el entorno y había que esperar para que hubiese comunicación, después de analizar la situación se llego a la conclusión de que había que establecer un algoritmo de sincronización que 
mejorara el desempeño de la comunicación en la plataforma. Para llevar a cabo tal fin se establecieron tareas en la cuales el robot maestro enviaba su mensaje mientras que el robot esclavo estaba en modo de recepción, cuando esto sucedía era el maestro quien entraba en modo de recepción y el esclavo enviaba su confirmación de recibido, todo esto dentro de un intervalo de 500ms (Fig. 11).

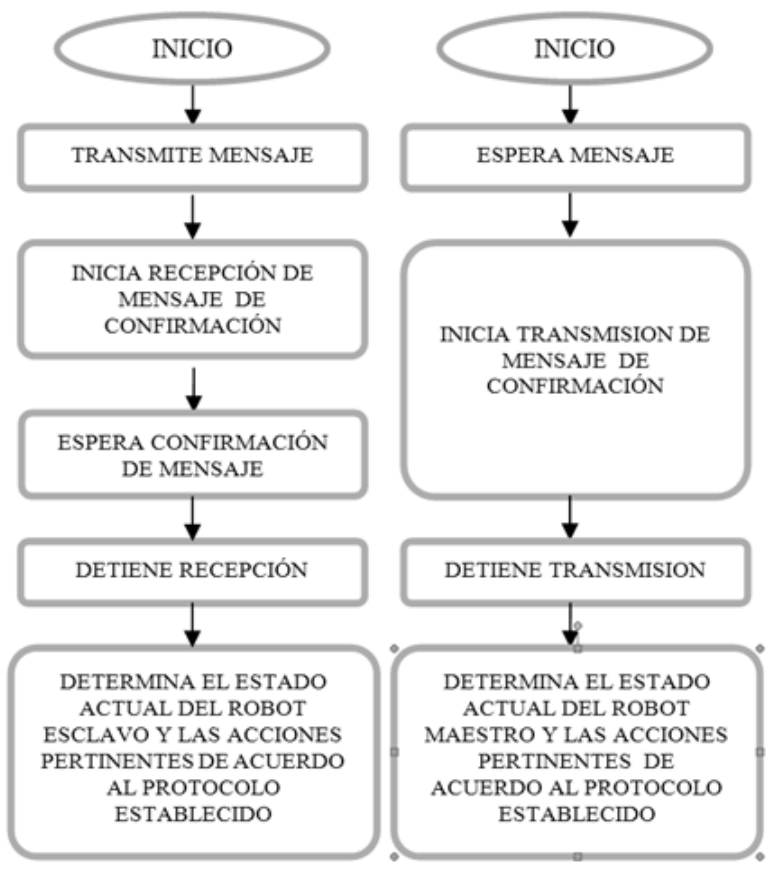

Fig. 11. Algoritmo de sincronización para la plataforma, a la izquierda se encuentra la lógica seguida por el robot maestro, a la izquierda la lógica seguida por el robot esclavo.

La comunicación de los robots se realizó mediante enlace infrarrojo y manejo caracteres en el rango de 0 a 255 (solo enteros), sin embargo se utilizaron siempre mensajes diferentes a cero ya que esta señal se podía confundir con una señal de ruido.

\section{RESULTADOS}

Después de todo el desarrollo del proyecto, la plataforma final contaba de un robot maestro (Fig. 12) y un robot esclavo (Fig. 13), cada uno con un dispositivo de sujeción de objetos.

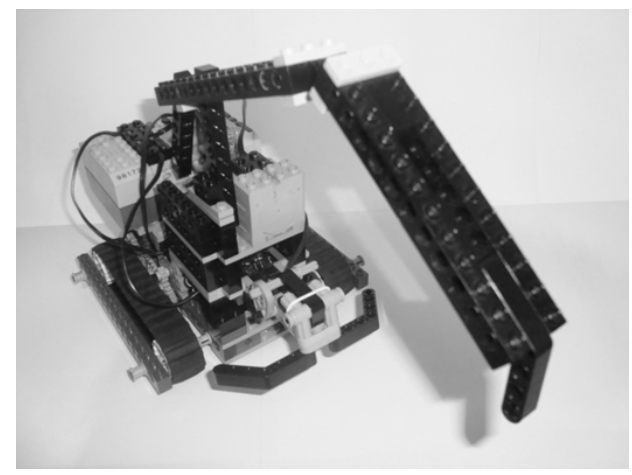

Fig. 12. Plataforma final (robot maestro)

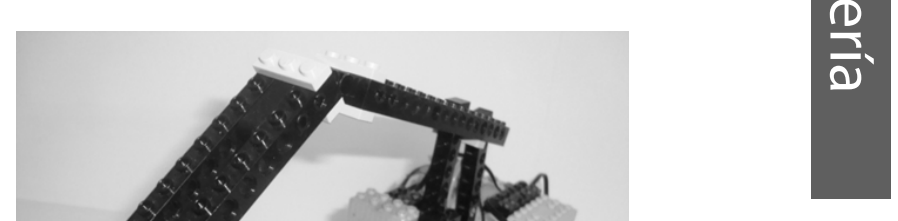

En la Fig. 14 se muestra el comportamiento del robot maestro en la fase de reconocimiento del objeto. La linea recta horizontal que simboliza la superficie del objeto, cuyo peso era de $3 \mathrm{Kg}$, en la grafica las variaciones por encima de esta linea representaron pequeños empujes que efectuó el robot al objeto mientras lo exploraba, por otra parte la variaciones por debajo de la linea horizontal significaron deficiencias del robot para llegar a tocar el objeto. Este comportamiento se altero mediante la implementación de algoritmos de corrección de ángulo los cuales a través de sencillas operaciones matemáticas efectuaban un control sobre el cambio de ángulo del robot disminuyendo la potencia de los motores cuando el ángulo registrado superaba los 90 grados y de la misma forma cuando el robot no alcanzaba un ángulo mínimo de giro se aumentaba la potencia de los motores para que pudiese llegar a tocar la superficie del objeto. 


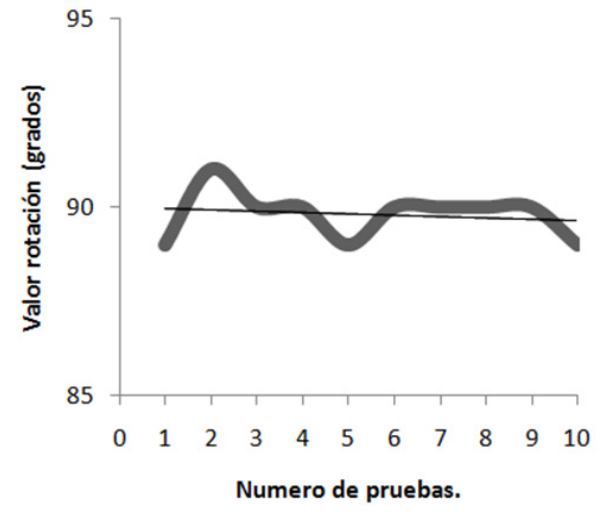

Fig. 14. Comportamiento del robot maestro en la fase de reconocimiento del objeto.

Para que los robots fueran en la misma posición al mismo tiempo se realizó una discretización de la posición del robot maestro, para tal fin se creó una tabla en donde se compararon las franjas blancas pintadas sobre el fondo de madera negro detectadas por los sensores de luz del robot esclavo junto con los valores medidos por el sensor de rotación del robot maestro de tal manera que la magnitud que representaba la posición del robot maestro se estaba referenciando a las franjas blancas (Fig. 15).

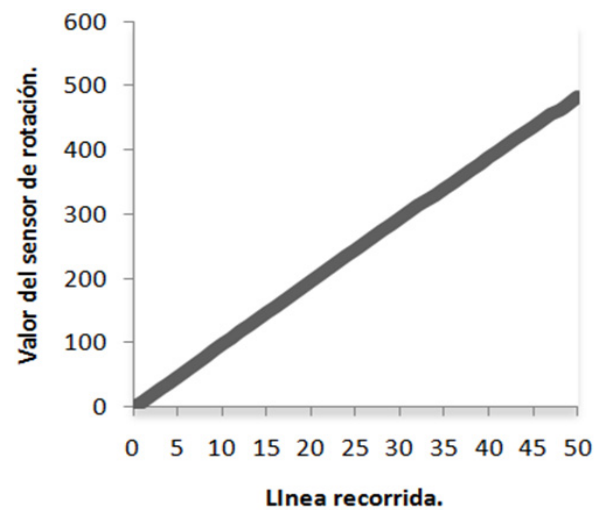

Fig. 15. Dicretización de la posición del maestro respecto a las líneas recorridas por el robot esclavo.

La relación entre las líneas recorridas por el robot esclavo y el valor otorgado por el sensor de rotación del robot maestro era una relación lineal, por lo cual se podía determinar una nueva relación, (Fig. 16) entre la distancia y las líneas recorridas por la plataforma donde se puede apreciar la correspondencia uno a uno entre las líneas y la distancia recorrida. Con esto se conocía exactamente la posición en cualquier lapso de tiempo.

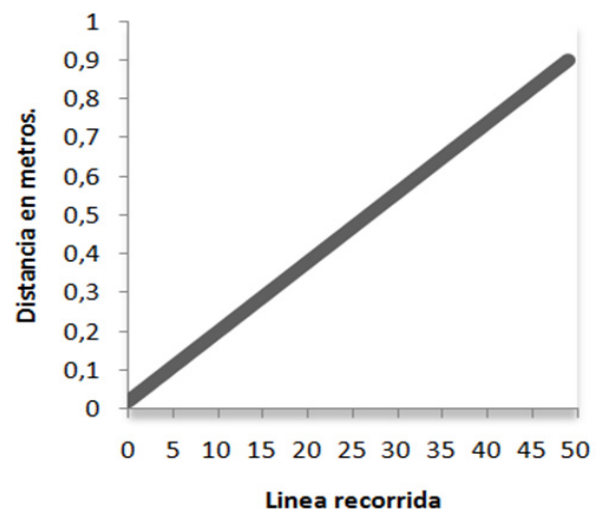

Fig. 16. Función línea recorrida vs distancia recorrida por la plataforma.

Fue necesario implementar una secuencia de comandos que evitara un cruce o pérdida de información, lo que permitió una adecuada interacción entre la plataforma y el entorno estructurado definiendo las acciones para cada uno de los robots. La secuencia de comandos se muestra en la tabla 1, a la derecha están las tareas del robot esclavo y a la izquierda las tareas del robot maestro.

Tabla 1. Secuencia de comandos para el establecimiento de las acciones en la plataforma.

\begin{tabular}{|l|c|c|}
\hline \multicolumn{1}{|c|}{ Comando } & $\begin{array}{c}\text { Robot } \\
\text { maestro }\end{array}$ & $\begin{array}{c}\text { Robot } \\
\text { esclavo }\end{array}$ \\
\hline Transporte cooperativo & 100 & 200 \\
\hline Transporte no cooperativo & 140 & 240 \\
\hline Arrastrar objeto & 110 & 210 \\
\hline Soltar objeto & 120 & 220 \\
\hline Regresar a la posición inicial & 130 & 230 \\
\hline Posición actual & $11-60$ & - \\
\hline Posición anterior & - & 70 \\
\hline
\end{tabular}

La plataforma transporto el objeto una distancia de un metro y presento una velocidad constante cercana a $0,14 \mathrm{~m} / \mathrm{s}$, la cual fue tomada desde el punto inicial hasta el punto final arrastrando objetos de $3 \mathrm{Kg}$ de peso (Fig. 17). 


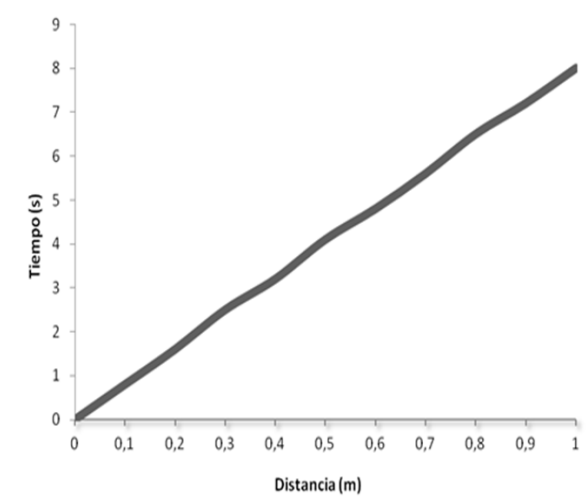

Fig. 17. Velocidad presentada por la plataforma durante el transporte del objeto.

\section{CONCLUSIONES}

Con el desarrollo de este proyecto continuaron las investigaciones en el campo de la robótica cooperativa dentro del grupo de investigación ROMA, generando bases para el posterior desarrollo de nuevas aplicaciones en este campo como búsqueda de objetos en un entorno desconocido, reconocimiento de objetos irregulares, y trabajo cooperativo que involucre un mayor número de plataformas.

La existencia de un entorno estructurado y conocido facilitó el desarrollo del proyecto en cuanto la posición espacial de la plataforma. Un posterior desarrollo de este proyecto podría ser en trabajo cooperativo en entornos poco estructurados.

\section{Referencias Bibliográficas}

[1] Pagina oficial de Lego Mindstorms. [En línea], consultado en Junio 10 de 2011, disponible en: http://www.lego.com.

[2] K. Guadarrama, A. López, Estrategias de coordinación para la exploración con multi-agentes roboticos, vol. Tesis para optar al titulo de Doctor, Universidad de puebla, Mexico, pp. 14-46, 2010.

[3] A. Marjovi, J. Nunes, L. Marques, A. de Almeida, Multi-robot exploration and fire searching, IROS'09 Proceedings of the
Se implemento una plataforma multi-robot autónoma que sirvió para el transporte de objetos de un punto a otro, dotada con los sensores y actuadores que ofrece el kit Lego Mindstorms RCX 2.0.

La interacción entre los robots de la plataforma se da de manera centralizada, donde el robot maestro procesaba toda la información y su robot esclavo actuaba como una extensión del primero, es decir que los objetivos se establecieron de manera global y cada una de las acciones de los robots contribuyo a la meta general del proyecto.

\section{FINANCIAMIENTO}

La robótica cooperativa es una de la líneas que se ha venido desarrollando dentro del grupo de investigación en Robótica Móvil Autónoma (ROMA), esta investigación se hizo en el marco del proyecto "diseño e implementación de agentes robots, actuando de manera cooperativa, en una zona colapsada simulada, como apoyo a organismos de búsqueda $\mathrm{y}$ rescate urbano".

\section{AGRADECIMIENTOS}

Al grupo de investigación en robótica móvil autónoma (ROMA) y en particular a su director MSc. Giovanni Rodrigo Bermúdez Bohórquez por su asesoría técnica y constante apoyo durante la ejecución de la investigación.

[4] M. J. Wooldridge, Intelligent Agents: in An introduction to multiagent systems ed: John Wiley \& sons Lda, 2002, pp. 15-42.

[5] M. Wooldridge and N. Jennings, Intelligent agents: Multiagent systems: A modern approach to distributed artificial intelligence, The MIT Press, 1999.

[6] A. Cervera, Coordinación y control 
de robots móviles basado en agentes, Escola Técnica Superior d'Enginyeria Informática, Universidad Politécnica de Valencia, Valencia España, 2011.

[7] G. Acosta, Ambiente multiagente robótico para la navegación colaborativa en escenarios estructurados, Tesis de Maestría, Facultad de Minas. Escuela de Sistemas, Universidad Nacional de Colombia., Medellín, Colombia., 2010.

[8] N. Vlassis, Chapter II. Rational Agents: in A concise introduction to multiagent systems and distributed artificial intelligence, Morgan \& Claypool Publishers, Ed., ed: Morgan \& Claypool Publishers, 2007, pp. 7-15.

[9] N. Jennings and M. Wooldridge, Applications of intelligent agents: Queen Mary \& Westfield College, pp. 1-10, 1998.

[10] Y. U. Cao, A. S. Fukunaga, and A. Kahng, Cooperative mobile robotics: Antecedents and directions, Autonomous robots, vol. 4, pp. 7-27, 1997.

[11] L. Parker, Chapter 40. Multiple Mobile Robot Systems: in Springer handbook of robotics, ed: Springer-Verlag New York Inc, 2008.

[12] C. Iglesias, Definición de una metodología para el desarrollo de sistemas multiagente, vol. Tesis Doctoral, departamento de Ingenieria de Sistemas Telematicos, Universidad Politecnica de Madrid, pp. 9-30, 1998.

[13] L. Iocchi, D. Nardi, M. Piaggio, and A. Sgorbissa, Distributed coordination in heterogeneous multi-robot systems, Autonomous robots, vol. 15, pp. 155-168, 2003.

[14] J. Jiménez, M. Valencia, and J. Ochoa, Metodología para el análisis y diseño de sistemas multi-agente robóticos: MADSmart, Medellin.Colombia, 2007.

[15] R. Rizo, F. Llorens, and M. Pujol, Arquitecturas y comunicación entre agentes, Departamento de ciencia de la computacion e inteligencia artificial,Universidad de Alicante, pp. 5-20, 2003.

[16] M. Mataric, M. Nilsson, and K. Simsarin, Cooperative multi-robot box-pushing, in in Proc. of the 1995 IEEE/RSJ Int. Conf. on Intelligent Robots and Systems, vol. 3. IEEE Computer Society Press, Los Alamitos, CA Los Alamitos, CA, 1995, pp. 556-561

[17] B. Donald, L. Gariepy, and D. Rus, Distributed manipulation of multiple objects using ropes, Proceedings of IEEE International Conference in Robotics and Automation, vol. 1, pp. 450-457, 2000.

[18] C. Kube and E. Bonabeaub, Cooperative transport by ants and robots, Robotics and Autonomous Systems, vol. 30, pp. 85$101,2000$.

[19] C. Kube and H. Zhang, Task modelling in collective robotics, Autonomous robots, vol. 4, pp. 53-72, 1997.

[20] Z. D. Wang and V. Kumar, Object closure and manipulation by multiple cooperating mobile robots, 2002, pp. 394-399 vol. 1.

[21] R. Gross, F. Mondada, M. Dorigo, Transport of an object by six pre-attached robots interacting via physical links, In Proc. of the 2006 IEEE Int. Conf. on Robotics and Automation, ICRA 2006, IEEE Computer Society Press, Los Alamitos, pp. 1317-1323, 2006.

[22] R. Gross and M. Dorigo, Cooperative Transport of Objects of different shapes and sizes, presented at the ant colony optimization and swarm intelligence, 4th International Workshop, ANTS 2004, volume 3172 of Lecture Notes in Computer Science, Berlin, Germany, 2004.

[23] F. Mondada, G. Pettinaro, A.Guignard, I. Kwee, D.Floreano, J.Deneubourg, S.Nolfi, L. Gambardella, and M. Dorigo, Swarm-Bot: a new distributed robotic concept, Autonomous robots, pp. 17(23):193-221, 2004.

[24] M. Dorigo, E. Tuci, V. Trianni, R .Gross, S. Nouyan, C. Ampatzis, T. Labella, R. O'Grady, M. Bonani, and F. Mondada, SWARM-BOT: Design and implementation of colonies of selfassembling robots.," Computational Intelligence: Principles and Practice, Gary Y. Yen and David B. Fogel (eds.), IEEE Computational Intelligence Society, 
pp. 103-135, 2006.

[25] M. Dorigo, D. Floreano, L. Gambardella, F. Mondada, S. Nolfi, T. Baaboura, M. Birattari, M. Bonani, M. Brambilla, and A. Brutschy, Swarmanoid: a novel concept for the study of heterogeneous robotic swarms, Technical Report TR/IRIDIA/2011-014, IRIDIA, Université Libre de Bruxelles, Brussels, Belgium2011.

[26] F. Ducatelle, G. Di Caro, and L. Gambardella, Cooperative selforganization in a heterogeneous swarm robotic system, In Proceedings of the Genetic and Evolutionary Computation Conference (GECCO), 2010.

[27] A. Decugniere, B. Poulain, A. Campo, C. Pinciroli, B. Tartini, M. Osée, M. Dorigo, and M. Birattari, Enhancing the cooperative transport of multiple objects, In Proceedings of the Sixth International Conference on Ant Colony Optimization and Swarm Intelligence (ANTS-2008), Springer, Lecture Notes in Computer Science, 2008, pp. 304-311.

[28] E. Ferrante, M. Brambilla, M. Birattari, and M. Dorigo, Look out!: Sociallymediated Obstacle avoidance in collective transport, In Proceedings of the Seventh International Conference on Swarm Intelligence (ANTS 2010), M. Dorigo and others (editors), Springer, vol. 6234, pp. 572-573, 2010.

[29] E. Ferrante, M. Brambilla, M. Birattari, and M. Dorigo, Socially mediated negotiation for obstacle avoidance in collective transport, in In 10th International Symposium on Distributed Autonomous Robotics Systems, 2010, To appear 2010.

[30] R. Gross and M. Dorigo, Towards group transport by swarms of robots, Int. J. BioInspired Computation, vol. 1, p. 1, 2009. 